\title{
Performance Analysis of the XDAC Disk Array System
}

\author{
Chiung-San Lee and Tai-Ming Parng \\ Rm. 350, Dept. of Electrical Engineering \\ National Taiwan University, \\ Taipei, Taiwan, R.O.C.
}

\begin{abstract}
This paper presenst an analytical model of a whole disk array architecture, $X D A C^{*}$, which consists of several major subsystems and features: (1) the twodimensional array structure, (2) IO-bus with split transaction protocol, and (3) cache for processing multiple $I / O$ requests in parallel. Our modelling approach is based on a subsystem access time per request (SATPR) concept, in which we model for each subsystem the mean access time per disk array request. The model is fed with a given set of representative workload parameters and then used to conduct performance analysis for exploring the impact of fork/join synchronization as well as evaluating some architectural design issues of the XDAC system. Moreover, by comparing the SATPRs of subsystems, we can identify the bottleneck for performance improvements.
\end{abstract}

\section{1: Introduction}

Disk array is emerging as an important class of disk I/O architectures [8]. The architecture consists of a group of disk devices, which are connected through several I/O buses to a disk array controller board (or a host adapter). The design of a disk array is complex, and prototyping the system is expensive. Thus, this class of systems represents an important opportunity for performance models to contribute to the actual design process.

The XDAC disk array system is a new twodimensional disk array architecture for providing high I/O bandwidth [5]. The first characteristic of the architecture is the ability to employ multiple disk devices in an array by striping data over them; i.e., the architecture has to maintain fork/join synchronization for disk array requests $[10,17]$. A disk array request is broken up into

* XDAC system is a prototyping disk array system developed in Industrial Technology Research Institute, Taiwan.

\author{
Jew-Chin Lee, Cheng-Nan Tsai, Kwo-Jean Farn \\ Industrial Technology Research Institute \\ Hsinchu, Taiwan, R.O.C.
}

independent physical disk requests (fork), all of which must complete to satisfy the disk array request (join). Another key characteristic of the architecture is that the organization combines multiple disk arrays connected by the I/O buses with split transaction protocol [15] to the disk array controller board; and thus the architecture may provide high $\mathrm{I} / \mathrm{O}$ bandwidth that is achieved by serving multiple disk array requests concurrently. Finally, the architecture is equipped with a cache placed on the $\mathrm{XDAC}$ board, called disk cache. If disk data is likely to be-referenced, the disk cache can be effective eliminating the disk seek and rotation, and improve system throughput. Therefore, in this architecture as shown in Fig. 1, several subsystems (e.g., disk cache, intelligent disk array controller, IO-bus, and disk device) are employed for obtaining high $\mathrm{L} / \mathrm{O}$ throughput.

Many previous works have conducted extensive performance analysis studies on disk array designs by using either simulation or analytical models $[2,4,6,7,9,10]$. Smith [7] developed the tracer driven simulation for exploring disk cache design considerations including cache size, cache location, block size, and so on. Lee [6] has derived a formula for the optimal size of data striping in disk arrays. Kim [9] employed the $M / G / 1$ queueing model to evaluate the impact of block sizes on system throughput and provide a guide that additional disks must be used to increase further the system throughput. However, these studies focused performance evaluation on each individual subsystem without addressing the whole disk array system and the relationship among subsystems.

In this paper, we present a performance model which has been used to study the performance prediction, several design consideration, and bottleneck analysis for the XDAC disk array architecture design. Our modelling approach takes a subsystem-oriented point of view and 
models the mean access time of each subsystem for the service of each disk array request to the XDAC system (called Subsystem Access Time Per Request, SATPR). From these subsystem access times, the response time of a disk array request and overall system throughput can be obtained. By comparing the SATPRs of the disk cache, IDAC, IO-bus, and disk device, the bottleneck can be identified. Then, we explore the effect of placing a cache at each disk device, called device cache, for removing the potential bottleneck. Furthermore, the model has been validated to be fairly accurate by statistical simulations which simulate the behaviors of disk array requests in the XDAC disk array system.

The rest of this paper is organized as follows. We describe the architecture of the XDAC disk array system in section 2. In section 3, we develop the subsystemoriented performance model of the target system. Section 4 describes the usage of the analytical model for evaluating system throughput and identifying the potential bottleneck. Section 5 contains the conclusions of the study.

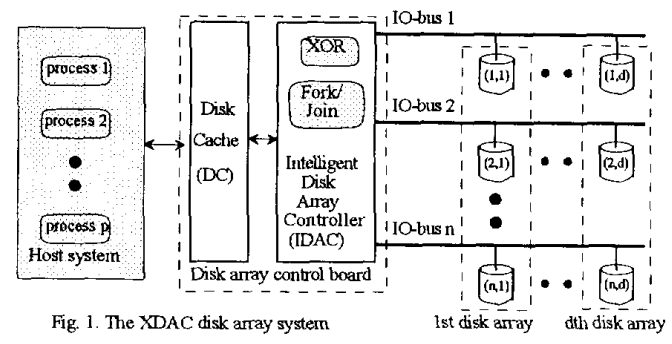

\section{2: The XDAC disk array architecture}

The XDAC disk array system consists of several subsystems, including disk cache, intelligent disk array controller (IDAC), IO-bus, and a group of disk devices for two-dimensional configuration as shown in Fig. 1. It serves the disk array requests from processes and issues physical disk requests to disk devices via the IO-bus.

The two-dimensional array organization is based on a grid of disk devices to support high I/O bandwidth. A single "column" of disk devices can be treated as individual devices for small disk array request, and as a disk array for large disk array request. In other words, any disk device can be accessed individually or as part of a disk array. Combining the concept of several disk arrays with IO-bus connecting to the IDAC yields the two dimensional array structure. A single "row" of disk devices that share an identical IO-bus is called a string. The key feature that must be supported in the string configuration is the ability to have overlapped seeks for serving multiple physical disk requests simultaneously. Therefore, the goal of the two-dimensional array organization is to maximize disk utilization and minimize the disk array request latency.

The disk cache serves as a buffer between the processes and disk devices. The disk cache is normally used to supply a disk data without accessing disk devices, when the data has been prefetched by previous requests, and then reduce the disk latency. If the disk cache is unable to supply the requested data, called disk cache miss, the IDAC will serve the disk cache miss request and start some of disk devices to access the data. In addition, the disk cache also eliminates disk time from the disk cache response to the disk write completion; i.e., a block of data is received from a process is initially written into disk cache and a completion message is sent to the process at that time. Data blocks in the disk cache that need to be written to disk devices are called dirty block. Such dirty blocks are written into disks we call destaging [12]. Actual destaging of data blocks from the disk cache into disk devices is done asynchronously at a later time. Thus, the disk devices only see the disk cache miss requests and destaging requests. Hereafter in this paper, we simple use the term "request" to represent the disk array request, disk cache miss request, or destaging request.

In addition to the above processing for different requests, the IDAC provides several special functions, such as fork/join synchronization and exclusive-OR (XOR) computation. Fork represents the simultaneous broadcast of several physical disk requests which are issued at the arrival of a disk cache miss request at the IDAC (these requests with family relationship are called family requests. Soon after, the time when all children have been serviced by the disks can be viewed as a virtual join synchronization between these family requests. In other words, a disk cache miss request is divided into a number of independent physical disk requests; all of them must complete to satisfy the disk cache miss request. Each physical disk request is serviced on a disk device 
concurrently with other physical disk requests. The XOR computation is used to generate a parity block for destaging some related data blocks $[1,8]$. When a block is been destaging to a disk, it is also necessary to update the parity block for a data stripe. This may require the IDAC to read the old values of the data block and the parity block from disks, to XOR them with the new values of the data block in the disk cache, then to write the new values of the data block and of the parity block to disk devices.

The IO-bus plays the role of the connection pathway for data transfer between the IDAC and all disk devices. The IO-bus is often standard bus (e.g., small computer standard interface, SCSI, bus [15]) that can have many devices connecting to it. The data transfer on an IO-bus consists of three phases, namely arbitration, selection, and information. The initiating (requesting) device first checks to see if the bus is free, and if so, then attempts to take control of it. If no higher priority device also bids for it, the initiator captures the IO-bus. During the selection phase, the initiator flags the target device with which it desires to communicate. The target device then responds with the type of transfer in which it is prepared to engage: data in/out, command request, and status acknowledge, during the information phase.

The IO-bus provides disconnect and reconnect functions based on split transaction protocol. These functions allow that the initiator releases the bus when it is not being used for transmitting the information. In other words, the IO-bus is available during the time that the target device services a command. Therefore, the functions make multiple devices to be active simultaneously which enhances bus utilization.

\section{3: Performance model of the XDAC system}

In this section, we present the modelling and analysis for each subsystem of the XDAC disk array system. Modelling the subsystem access time for the XDAC system, performance metrics, such as response time, throughput, SATPR, partial utilization, partial queue length, and waiting time, of each subsystem will be derived.

We use the notation presented in Table I for modelling the XDAC system, including request types and input parameters. From Table I, we can see that the request types of the four subsystems include dar, dcm, dtg, chld requests. The request type, dar, will visit the disk cache; this type request will block the requesting process, until the returned data is received. Two request types, including $\mathrm{dcm}$, dtg, visit the IDAC, IO-bus, disk device subsystems; only the $d \mathrm{~cm}$ request will cause the requesting process to be blocked. In other words, the dar and $\mathrm{dcm}$ requests belong to blocking requests; the $d t g$ request can be asynchronously processed and its acknowledge is replied to the requesting process.

(I) Service Time: Most service times of subsystems are deterministic, but some are request- or utilization-

Table I

Notation for Performance Modelling of the XDAC System

Request Types:

- $H_{c a c h e}=\{d a r\}, H_{i d a c}=\{d c m, d t g, c h l d\}, H_{i o b u s}=H_{d i r k}=\{d c m, d t g\}$ denote the sets of request types that visit the disk cache, IDAC, IO-bus, and disk device.

- $B_{c a c h e}=\{d a r\}, B_{i d a c}=B_{\text {iobus }}=B_{\text {dist }}=\{\mathrm{dcm}\}$ denote the sets of blocking request types that visit the disk cache, IDAC, IO-bus, and disk device.

Input Parameters:

- $n$ is total number of disk devices in a disk array.

- $d$ is total number of disk arrays.

- $p$ is total number of processes in host system.

- $b$ is the number of data blocks for a dar requests.

- $f$ is the number of bytes of a track.

- $s$ is the number of bytes of a data block.

- $p_{d c m}$ is the disk cache miss rate; $p_{d t g}$ is the destaging probability.

- $t_{p}$ is the mean processing time of a process in the host.

- $t_{\text {cache }}$ is the mean access time of the disk cache.

- $t_{\text {idac }}$ is the processing time of the IDAC.

- $t_{x o r}$ is the XOR computation time for a data block.

- $t_{\text {connect }}$ is the time required to connect a pathway between a disk device and the IDAC via an IO-bus, including the arbitration time and the selection time.

- $t_{\text {posi }}$ is the average time of a disk device to position the access head to the cylinder containing the data.

- $t_{i 2 \text { s }}$ is the average seek time of track to track.

- $t_{\text {revo }}$ is the average revolution for a disk device.

- $t_{\text {buff }}$ is the average time required to read/write a data block from/to disk surface.

- $t_{\text {traf }}$ is the average transferring time via an IO-bus.

The output parameters are defined in the development of the equations. 
dependent. The service time of the disk cache which is assumed to be constant is denoted as $t_{\text {cache }}$ for a data block. The service time of the IDAC is requestdependent on the request types; the service times of $\mathrm{dcm}$ and $d t g$ requests in the IDAC are the $t_{i d a c}$ and $\left(t_{i d a c}+t_{x o r}\right)$, respectively, for a data block.

The free time of the IO-bus is dependent on their own utilization and can be derived as follows: let $U_{\text {iobus }}$ denote the partial utilization of an IO-bus raised by the requests from a particular process; $p U_{\text {lobus }}$ is the total utilization of an IO-bus used by $p$ processes. A new request from the other $p-1$ processes can be issued during the remaining part of time (when the IO-bus is not busy), i.e., (1- $U_{\text {iobus }}$ ). Thus, ( $\left.p U_{\text {iobus }}-U_{\text {iobus }}\right)$ is the partial utilization of the IObus taken to serve the requests from other processes. The probability that the IO-bus is idle (i.e., ready for a new request $)$ is $\left[1-\left(p U_{\text {iobus }}-U_{\text {lobus }}\right) /\left(1-U_{\text {iobus }}\right)\right]$.

The service time of an IO-bus includes the time to connect the pathway and the time to transfer a data block between the disk cache and a disk device. When a disk device is ready to transfer a block data, the disk attempts to reconnect the IO-bus [15]. If the IO-bus is free, this reconnect succeeds and data transfer takes place. If the IO-bus is busy, reconnect attempts are continued until success is achieved. We assume that for any a particular disk device, the probability of failure on various reconnect attempts are independent. Let $P_{\text {success }}$ denote that the probability of a reconnect attempt of a disk device succeeds. Then, the IO-bus service time to transfer a data block can be estimated as

$$
\begin{aligned}
S_{\text {iobus }}= & t_{\text {traf }}+t_{\text {connect }}\left[1+\sum_{k=1}^{\infty}(k-1) P_{\text {success }}\left(1-P_{\text {success }}\right)^{k-1}\right] \\
& =t_{\text {traf }}+t_{\text {connect }} / P_{\text {success }}
\end{aligned}
$$

A reconnect attempt succeeds if the IO-bus is free, and fails otherwise; i.e., $P_{\text {success }}$ is equal to the probability of the IO-bus is free; i.e., $P_{\text {success }}=$ $\left[1-\left(p U_{\text {tobus }}-U_{\text {tobuen }}\right) /\left(1-U_{\text {iobus }}\right)\right]$.

The disk device service time, $S_{\text {disk }}$, includes three major components: seek time, rotational latency, and data transferring time. Seek time, $t_{\text {seek }}$, is the sum of the time required to position the access head to the cylinder that contains the requested data, and the time to position the access head to the next cylinder when requesting size is larger than track size. Since the seek time is not linearly proportional to the distance the access arms travel and those disk arms often do not move for $50 \%$ to $70 \%$ of requests [9], we take a constant delay as the mean seek time which can be estimated as

$$
t_{\text {seek }}= \begin{cases}t_{\text {part }} & \text { if } s \leq f \\ t_{\text {past }}+\frac{\lceil s\rceil}{f} t_{t 2 t s} & \text { otherwise. }\end{cases}
$$

Rotational latency, $t_{\text {rota }}$, is the time required for the correct data or track area of the disk to rotate under the access head; it can be ranged from zero to full revolution of the disk. In general, half the rotational time is used as the average latency $[2,3,9,10]$.

$$
t_{\text {rota }}= \begin{cases}t_{\text {revo }} / 2 & \text { if } s<f \\ 0 & \text { otherwise. }\end{cases}
$$

The data transferring time, $t_{b u p p}$ is the time required to read (or write) a data block from (or to) the disk device's surface. Therefore, the disk service time can be estimated as

$$
S_{\text {disk }}=t_{\text {seek }}+t_{\text {rota }}+t_{\text {buff }}
$$

In above equation, we assume that each disk device is equipped with a track buffer for eliminating the rotational positioning sensing (RPS) miss.

(2) Response Time: The mean response time of a type $j$ blocking request to the $i$ th subsystem, $R_{i, j}$, is dependent on the waiting time caused by those requests that is currently queued or being served in the subsystem and on the service time of the blocking request itself and can be estimated as

$$
R_{i j}=\sum_{k \in H_{j}} W_{i j, k}+S_{i} ; \quad j \in B_{i}
$$

where $i \in\{$ cache, idac, iobus, disk $\}$ and $\sum_{k \in H_{1}} W_{i j, k}$ is the mean waiting time of a blocking request of type $j$ at the $i$ th subsystem caused by all request types in $H_{i}$.

(3) Subsystem Access Time Per Request (SATPR): The SATPR of the $i$ th subsystem is the sum of the response 
times of all blocking requests from a particular process and can be estimated as

$$
T_{i}=\sum_{j \in B_{i}} p_{j} R_{i j} \quad i \in\{c a c h e, i d a c, i o b u s, \text { disk }\}
$$

where $p_{j}$ is the probability of the type $j$ requests to visit the $i$ th subsystem.

The mean subsystem access time per request at a disk device and an IO-bus, $T_{s d}$, is estimated as

$$
T_{s d}=p_{d c m}\left(R_{\text {iobus,dcm }}+R_{d i s h, d c m}\right) \text {. }
$$

(4) Fork/Join synchronization: The disk cache miss $(\mathrm{dcm}$ ) and destaging ( $\mathrm{dtg}$ ) requests will be forked to a number of physical disk requests; each physical disk request is being serviced by an IO-bus and a disk device concurrently with other physical disk requests. When these family of physical disk requests are complete, they must join in together

Based on a scaling approximation technique proposed by Nelson and Tantawi [17], we can compute the fork/join synchronization time for these family of $b$ physical disk requests. Since the fork/join synchronization time for several family requests is caused by the $T_{s d}$, the mean synchronization time for two family requests is given by $T_{s y n c, 2}=\left(0.5-0.125^{*} \rho\right)^{*} T_{s d}$, where $\rho=T_{s d} / T_{p r o}$. Therefore, the fork/join synchronization time of $b$ family physical disk requests, $T_{s y n c}$, can be estimated as

$$
T_{\text {sync }}= \begin{cases}0 & \text { if } b=1 \\ {\left[\frac{H_{b}}{H_{2}}+\frac{4}{11}\left(1-\frac{H_{b}}{H_{2}}\right) \rho\right]^{*} T_{s y n c, 2}} & \text { otherwise }\end{cases}
$$

where $H_{b}$ is harmonic series and $H_{b}=\int_{0}^{\infty}\left(1-\left(1-e^{-t}\right)^{b}\right) d t$.

(5) Average processing time per disk array request: The average processing time per disk array request seen by process, $T_{d a r}$, eqauls to the sum of all SATPRs in each subsystem and of the synchronization time, $T_{s y n c}$, and can be expressed as

$$
T_{\text {dar }}=T_{\text {cache }}+T_{\text {idac }}+T_{\text {iobus }}+T_{\text {disk }}+T_{\text {symc }}
$$

The mean time between two successive disk array requests from the same process, $T_{p r o}$, is the sum of execution time of a process in the host, $t_{p}$, and of $T_{d a r}$, i.e.,

$$
T_{p r o}=t_{p}+T_{d a r} .
$$

(6) Partial Utilization: The partial utilization of the $i$ th subsystem used by any request of type $k$ to request $b$ data blocks from a particular process, $U_{i, k}$, is the ratio of the service time by the requests over total elapsed time, i.e., $T_{p r o}$, and can be estimated as

$$
U_{i, k}=p_{k} S_{i} / T_{p r o}
$$

where $i \in\left\{\right.$ cache, idac, iobus, disk\}, $\quad k \in H_{i}, \dot{p_{k}}$ is the probability that the request is of type $k$, and $S_{i}$ is the $i$ th subsystem service time.

(7) Queue Length: The average partial queue length of the $i$ th subsystem includes the average number of requests that is currently being served by the subsystem. Therefore, the mean partial queue lengths of requests of type $j$ at the $i$ th subsystem, $Q_{i, j}$, by a particular process can be approximated by

$$
Q_{i, j}=p R_{i, j} / T_{p r o}=p_{j}\left(\sum_{k \in H_{i}} W_{i, k}+S_{i}\right) / T_{p r o}
$$

where $i=\{$ cache, idac, iobus, disk $\}, j \in H_{i}$.

(8) Waiting Time: An arriving request at the $i$ th subsystem will wait for the mean residual service time, i.e., mean remaining service time, of the request in service, plus one mean subsystem service time for every other requests in the queue when it arrives. We assume that the service time at each subsystem is deterministic; so the residual service time is approximately one-half its service time. Thus the waiting time of a request of type $j$ at the $i$ th subsystem caused by a request of type $k$, for $i=\{$ cache, idac, iobus, disk $\}$, and $j, k \in \mathrm{H}_{1}$, is

$$
W_{i, j, k}=\left\{\begin{array}{l}
(p-1)\left(\left(Q_{i, k}-U_{i, k}\right) S_{i, k}+U_{i, k} S_{i, k} / 2\right) ; \quad \text { if } j=k \\
p\left(\left(Q_{i, k}-U_{i, k}\right) S_{i, k}+U_{i, k} S_{i, k} / 2\right) ; \text { otherwise }
\end{array}\right.
$$

(9) Total Utilization: The partial utilization of the ith subsystem by a particular process is $U_{i}$ and can be estimated as

$$
U_{i}=\sum_{k \in H_{i}} U_{i, k} \quad i=\{\text { cache, idac, iobus, disk }\}
$$


The total utilization of the $i$ th subsystem used by $p$ processes is computed as $p^{*} U_{i}$.

(10) Overall Throughput: The overall throughput of the $\mathrm{XDAC}$ disk array system requested by $p$ processes is estimated as $\left(p b s / T_{p r o}\right)$ bytes.

(1I) Bottleneck Analysis: In addition to performance evaluation described above, the other important application of the SAT modelling methodology is to identify the potential bottlenecks existing in the XDAC disk array system. From Equation (3), we observe that the relative contribution of the various SATPRs to the overall system throughput, which is equal to $\left(p b s / T_{p r o}\right)$ bytes as described above, is immediately obvious. In other words, a subsystem with the longest SATPR is the one that limits the system throughput and creates a bottleneck to the XDAC disk array system. Note that the fork/join synchronization time is considered in the IDAC subsystem. Particularly, a large difference between subsystem's SATPRs is a symptom indicating existence of a bottleneck. Since a disk array system has at least one bottleneck, we can find that the $i$ th subsystem is a bottleneck, if

$$
T_{i}=\max \left\{T_{\text {cache }}, T_{\text {idac }}, T_{\text {iobus }}, T_{\text {dist }}\right\} .
$$

\section{2: Validating the SAT model of XDAC system}

A statistical simulation uses probability distributions to characterize the workload parameters. Since simulation languages or tools always offer a rich choice of such probability distributions, they can be used to generate input data to the computer system simulation model. When the simulation model is compiled, the input events are generated according to the programmed probability distributions. We develop a statistical simulation model of XDAC system by using SES/workbench [16] to obtain average process time per disk array request and validate the results from our analytical model.

To validate the XDAC model, we compare the performance estimates, $T_{d a r}$, from the mean value equations with estimates obtained from statistical simulations. To obtain some estimates, we consider several configurations with varying number of disk arrays, disk cache miss rates, and so on about 120 cases and the results are presented in Section 4. Fig. 2 shows the percentage differences in mean processing time per disk array request between the analytical model and the simulation. Since our emphasis in this paper is the values of the SATPR, we consider only the validity of the analytical model in all SATPRs. By comparing the results obtained from the both, we find that in about $91 \%$ of all cases the percentage difference is less than $3 \%$. We also find that in less than $1 \%$ of all cases the magnitude of the percentage difference is greater than $5 \%$.

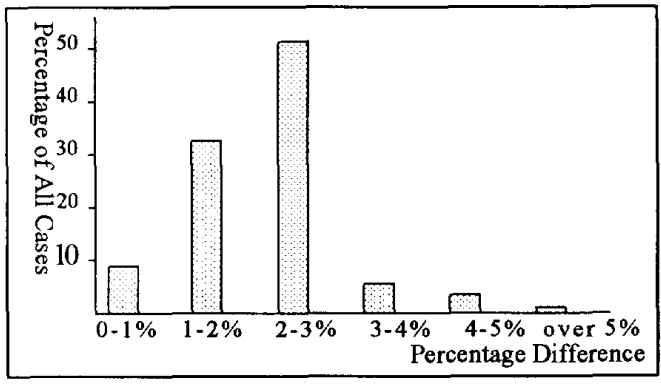

Fig. 2. Percentage difference between the results of analytical model and of simulation

\section{4: Evaluating the XDAC system}

In this section, we evaluate the performance of the proposed two-dimensional disk array architecture, XDAC system, by using the analytical model. We will describe the workloads for feeding the analytical model and discuss in detail performance prediction and bottleneck identification for the XDAC system. Also, we will explore the effect of placing a cache at each disk device for removing the bottleneck and the impact of the following performance influencing factors: number of disk arrays and fork/join synchronization time.

\section{1: Workloads for the Analytical Model}

We take most of the workloads for the analytical model of the XDAC system from existing literature. Many papers [7, 13] published some architecturedependent parameters. For examples, Smith [7] collected a variety of miss rates for various disk cache size; 
Friendman [13] observed that destaging requests are about one-fifth to one-third of all disk array requests. To provide input parameters to the SAT modelling, we obtain the values of $p_{d c m}, p_{d t g}$, and the miss rate of the device cache, from these papers that are representative of the workload. In general, the disk cache miss rate is inversely proportional to the cache size. Based on the results of Smith's collections, the disk cache size of 16 Mbytes has an average miss rate of $17 \%$ and the device cache size of 1 Mbytes provides an average miss rate of $54 \%$. The parameters of disk devices are based on the HP79560 disk [12]. In addition, we assume that there is five disk devices in a disk array across five IO-buses; each IO-bus connects $d(=1, \ldots, 8)$ devices in a string (i.e., $d$ disk arrays in the XDAC system).

\section{2: Throughput prediction and bottleneck identification}

Our SAT modelling can serve to predict the system throughput and identify potential bottlenecks. We obtain the system throughput by computing the average processing time per disk array request, as the number of processes and the number of disk arrays are varied. Fig. 3 shows these results. For the XDAC system with 16 Mbytes disk cache and 5 Mbytes transfer rate of each IO-bus, the system throughput increases from $18 \mathrm{Mby}-$ tes/sec with 1 disk array to 30 Mbytes/sec with 2 disk arrays, an improvement of $48 \%$. However, as we can see, the throughput increases about $6 \%$ from 2 disk arrays to 3 disk arıays. Therefore, there exists a bottleneck in the XDAC disk array system. In other words, we are unable to improve the performance of the XDAC system by only increasing the number of disk arrays.

For bottleneck identification, we compute the SATPRs for each subsystem. Fig. 4 shows the results with different number of processes. In every case, the SATPR of the disk device is the largest among these subsystems including the disk cache, IDAC, IO-bus, disk device, and fork/join synchronization. Since the increase of the SATPR in the disk device subsystem with varying number of processes keeps being the largest, the bottleneck must be present in the disk device subsystem.

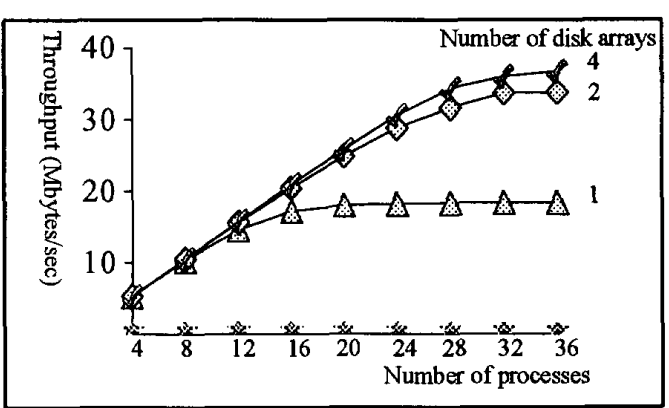

Fig. 3. System throughput v.s. number of processes

From Fig. 4, we also find that the fork/join synchronization time is also long and is the second factor that reduces throughput. The synchronization time is formulated by using the SATPRs of the IO-bus and disk device; i.e., a large SATPR of disk device will cause a long synchronization time. Therefore, by improving disk subsystem performance can also reduce the synchrounization time, as shown in Fig. 5. However, the impact of synchronization time on the $T_{d a r}$ is still large. From Fig. 4 and 5 , the $T_{\text {sync }} / T_{d a r}$ is about one-third; thus we believe that the impact of synchronization time will be significant and should be included in the performance model of a disk array system.

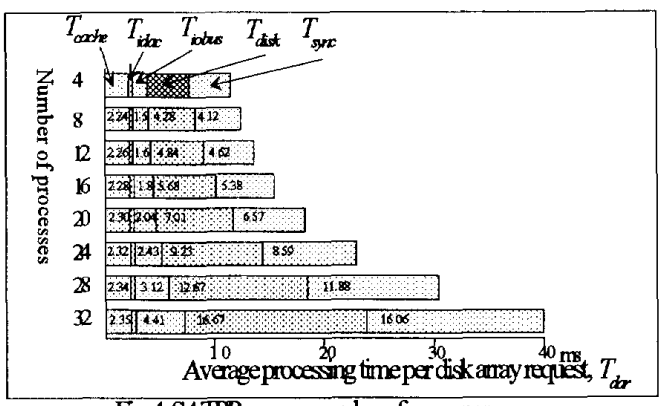

Fg. 4.SATPRsversusnmber of processes

\section{3: Impact of Placing Cache on each Disk Drive}

The bottleneck in the disk device subsystem can be removed by placing a device cache on each disk. Some of physical disk requests can be served by the device cache without accessing the disk device; thereby, the disk waiting time and service time are reduced. As we can see in the Fig. 5, the SATPR of disk device has been 
reduced by using the device cache. By comparing Fig. 4 with 5 , we find that using the device cache is effective method for improving the performance in the disk device subsystem.

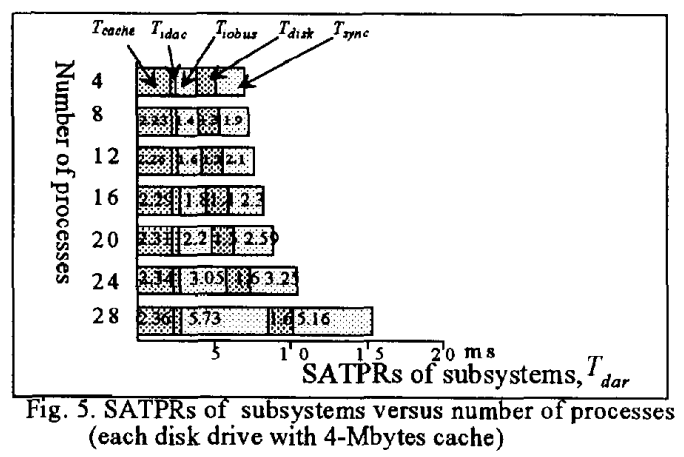

\section{5: Conclusion}

We have developed an analytical model for the XDAC disk array system which features of twodimensional disk array for serving multiple disk array requests in parallel, IO-bus with overlap of bus phases and disk device mechanism activities for increasing bus utilization, and disk cache to eliminate disk latency for increasing system throughput. Our modelling methodology is a divide-and-conquer approach; we divide a large disk array. system into small subsystems including disk cache, disk array controller, 1O-bus, and disk device, and model independently the subsystem access time for each individual subsystem. In addition to performance prediction, the subsystem access time per request (SATPR) of all subsystems can also be used for identifying which subsystem is the bottleneck and then exploring different configurations for potential performance improvements. Also, we have employed the model to investigate system design choices including number of disk arrays and cache size of each disk device.

\section{References}

[1] D. Patterson, G. Gibson, and R. Katz, "A case for redundant arrays of inexpensive disk (RAID)," ACM SIGMOD conference proceedings, pp. 109-116, Tune, 1988
[2] P.M. Chen, G.A. Gibson, R.H. Katz, and D.A. Patterson, "An evaluation of redundant arrays of disks using an Amdahl 5890," ACM Architecture News, pp 74-85, 1990

[3] A.L. Narasimha, P. Banerjee, "Design, Analysis, and Simulation of I/O Architectures for Hypercube Multiprocessors," IEEE Trans. on parallel and distributed system, pp.140-151, Apr. 1990.

[4] A.L. Narasimha, P. Banerjee, "An Evaluation of multiple-disk I/O systems," IEEE Trans. on Computers, pp. 1680-1690, Dec. 1989.

[5] ITRI, CCL, XDAC architecture

[6] P.M. Chen and D.A. Patterson, Maximizing Performance in a striped disk array," International Symposium on Computer Architecture, May 1990.

[7] A.J. Smith, "Disk cache: Miss ratio analysis and design considerations," ACM Trans. Comput. Sys., vol. 3, no. 3, pp. 161-203, Aug. 1985

[8] R.H. Katz, G.A. Gibson, and D.A. Patterson, "Disk System architectures for high performance computing," Proceedings of the IEEE, vol. 77, no.. 12, Dec. 1989

[9] M. Kim, "Synchronized Disk Interleaving," IEEE T. on Comput. vol. C-35, no. 11, Nov. 1986

[10] E.K. Lee and R.H. Katz, "An Analytic Performance Model of Disk Arrays," ACM SIGMETRICS, 1993

[11] D. Stodolsky, G. Gibson, and M. Holland, "Parity Logging Overcoming the small Write problem in Redundant Disk Arrays," ACM Architeqture News, 1993

[12] J. Menon and J. Cortney, "The Architecture of a FaultTolerant Cached RAID Controller," $A C M$ Architecture News, 1993

[13] M.. Friendman, "DASD access patterns," In Procedings of the 1983 CMG Intern. Conference, pp. 51-61, 1983

[14] M.Y. Kim, "Synchronized Disk Interleaving," IEEE T. on Comput., vol. c-35, no. 11, Nov. 1986

[15] D.A. Patterson and J.L. Hennessy, "Computer Organization \& Design - the hardware / software interface," Morgan Kaufmann, 1994

[16] SES/workbench User's manual and Reference's manual Release 2.1, Feb. 1992.

[17] R. Nelson and A. N. Tantawi, "Approximate analysis of Fork/Join Synchronization in Parallel Queues," IEEE $T$. Comput. vol. 37, no. 6, June 1988 